\title{
A value-based evaluation of minimally invasive hysterectomy approaches
}

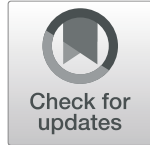

\author{
Natalya Danilyants ${ }^{1}$, Paul MacKoul', Louise van der Does ${ }^{1 *}$ (D) Leah Haworth ${ }^{1}$ and Rupen Baxi ${ }^{2}$
}

\begin{abstract}
Background: With surgical care contributing to the rising healthcare costs around the globe, quantifying the value of surgical modalities is critical to pushing healthcare systems in the direction of greater sustainability. The purpose of this study was to assess and compare the value of minimally invasive hysterectomy approaches, as defined by operative outcomes and patient satisfaction relative to direct hospital costs.

Methods: Sequential mixed methods; retrospective chart review of all women $(N=2689) \geq 18$ years old who underwent hysterectomy for benign conditions from 2011 through 2013 at a suburban hospital in Maryland, USA; a mail survey of the same population was administered in June to October 2015. Procedures included laparoscopic supracervical hysterectomy (LSH), robotically assisted laparoscopic hysterectomy (RALH), total laparoscopic hysterectomy $(T L H)$, laparoscopically assisted vaginal hysterectomy ( $L A V H)$, total vaginal hysterectomy $(T V H)$, and laparoscopic retroperitoneal hysterectomy $(\mathrm{LRH})$, a procedure that incorporates retroperitoneal dissection and ligation of the uterine arteries at their vascular origin. We estimated the value of each procedure by dividing quality over direct hospital costs, where quality was quantified by creating a summary composite score of the average operating time, estimated blood loss, complication and conversion rates, and patient satisfaction.
\end{abstract}

Results: The majority of LRH and RALH procedures were performed by high-volume surgeons, while the majority of LSH and TVH procedures were performed by low-volume surgeons. RALH had the highest postoperative complication rate $(11.4 \% ; p<.0001)$. TVH had the highest intraoperative complication rate $(9.6 \% ; p<.0001)$ but lowest postoperative complication rate $(1.8 \% ; p<.0001)$. LRH had the shortest operating time $(71.2 \mathrm{~min} ; p<.0001)$ and lowest intraoperative complication rates $(2.1 \% ; p<.0001)$. LRH and TVH were the least costly $(\$ 4061$ and $\$ 6416$, respectively), while RALH was the most costly (\$9354). LRH had the highest combined patient satisfaction score, followed by RALH, while TLH, LAVH, TVH, and LSH averaged similar scores.

Conclusion: $L R H, T V H$, and $L A V H$ yielded the highest value scores; $L S H, T L H$, and RALH yielded the lowest. Healthcare costs continue to escalate, in large part due to innovations in medical technology. For healthcare stakeholders seeking to control costs without sacrificing quality, it is critical to operationalize the value of varying surgical techniques, including measures of surgeon experience, operative outcomes, costs, and patient satisfaction.

Keywords: Cost, Hysterectomy, Laparoscopic, Retroperitoneal, Value, Satisfaction

\section{Background}

Hysterectomy remains the most common non-obstetric surgical procedure for women in the USA and accounts for more than $\$ 5$ billion in healthcare spending [1]. The skyrocketing costs of healthcare have intensified the focus on value-based healthcare delivery. While much of the conversation, study, and policy shifts have centered

\footnotetext{
* Correspondence: Lvanderdoes@towersurgicalpartners.com

${ }^{1}$ The Center for Innovative GYN Care, 3206 Tower Oaks Blvd., Suite 200,

Rockville, MD 20852, USA

Full list of author information is available at the end of the article
}

around outcomes and costs, it is imperative that these value assessments also consider the experience of the most central player in the healthcare system: the patient [2].

The patient-centered model has gained considerable attention over the past decade. Today, there are several well-established agencies that address continuous quality improvement using patient-centered management processes to assess the quality of healthcare. For example, the Centers for Medicare and Medicaid Services (CMS), 
the largest payer in the USA, has shifted from fee-for-service to pay-for-performance in an effort to reward hospitals for quality care [3]. CMS created the Value-Based Purchasing program, which withholds up to $2 \%$ of reimbursements to hospitals annually, with the opportunity to earn back these reimbursements based on the hospital's performance score for the fiscal year. Significantly, $25 \%$ of these hospital performance scores are based on patient satisfaction [3]. Thus, poor performance on patient satisfaction metrics may represent a substantial financial risk for hospitals.

In 2014, the National Quality Forum sponsored the white paper, "Efficiency and Value in Healthcare", reviewing various approaches that combine quality and cost indicators to measure value [4]. Ryan and Tompkins and Timbie and Normand describe the "conditional model" in which quality is assessed by a single indicator or a composite measure, and cost is assessed by a single measure of total costs $[4,5]$. Both the quality and cost domains are classified into performance groups using specified criteria such as "low," "average," or "high." Those falling under high-quality and low-cost tiers are typically considered high-value providers $[4,5]$. A variation of the conditional model adds the hurdle of reaching either a minimum quality or cost standard before the performance is evaluated [6]. The "unconditional model" follows the aforementioned steps, but the quality and cost parameters are assigned weights and collapsed into a single metric [4]. This is the model used by CMS' Hospital Value-Based Purchasing program [3]. Timbie and Normand and Kessler and McClellan also put forth a cost-effectiveness model using a weighting system for desirable health outcomes relative to the absolute cost $[5,7]$.

Additionally, more and more patients seek doctors based on online reviews, ratings, and social media-driven recommendations. Patients are gathering information and evaluating doctors even before presenting to the office for an initial visit [8]. Thus, patient satisfaction has become an important element in the concept of value from the perspective of the consumer and is helping to drive the economics of healthcare [9]. Cowing underscores this concept: "[A]s health care competitive dynamics continue to evolve, it is no longer sufficient to define health care performance in terms of clinical outcomes alone. The inclusion of patient satisfaction is fast becoming an important dimension because the notion of consumer-driven health care increasingly applies to patient choice in the health care industry" [10].

While the need to use cost and quality measures to assess the value of physician practices, hospitals, and health plans is well established, there is currently no consensus or practical set of guidelines on how to compare the value of surgical modalities. As healthcare costs continue to escalate, in large part due to innovations in medical technology, operationalizing the value of varying surgical routes of hysterectomy is critical for healthcare stakeholders seeking to control costs without sacrificing quality. As Wright et al. point out: "With changing technology and further cost-minimization strategies, a shift must occur toward cost-effective minimally invasive methods of hysterectomy, so the health care system can experience more savings and greater clinical excellence with regards to the most common gynecologic procedure performed" [11].

Our study attempted to assess and compare the value of the most common minimally invasive hysterectomy procedures by using a conceptual model of three interrelated criteria: operative outcomes, patient satisfaction, and costs.

\section{Methods}

We conducted a retrospective chart review of 2689 patients, 18 years and older, who underwent minimally invasive hysterectomy between January 2011 and December 2013 at a not-for-profit, high-volume hospital, located in suburban Maryland serving the greater metropolitan Washington, DC area. The surgeons included obstetrician/gynecologist generalists and laparoscopic surgeons trained in various gynecologic fellowships such as minimally invasive surgery, gynecologic oncology, and urogynecology. This investigation was approved by the hospital's Institutional Review Board (IRB).

Cases with concomitant procedures frequently performed during a hysterectomy, such as adnexal removal, adhesiolysis, and cystoscopy, were included in the analysis. Patients with malignant indications for hysterectomy and cases with major concomitant procedures unrelated to the hysterectomy, such as bowel resection, appendectomy, cholecystectomy, hernia repair, and major pelvic support procedures, were excluded from review to avoid artificially inflating costs. Surgeon volume, as defined by the number of hysterectomy cases performed per year by individual surgeons, was also collected and recorded in order to control for surgeons' skill and experience.

Previous studies comparing minimally invasive gynecologic techniques relied solely on Current Procedural Terminology (CPT) and the International Classification of Diseases, Ninth revision (ICD-9) diagnostic codes to classify gynecologic procedures [11-13]. In order to increase the validity and reliability of our data, we confirmed and corrected the coding schema by reviewing each operative report. Hysterectomy procedures were originally identified and classified on the basis of the ICD-9 diagnostic codes for laparoscopic supracervical hysterectomy (LSH), robotically assisted laparoscopic hysterectomy (RALH), total laparoscopic hysterectomy 
(TLH), laparoscopically assisted vaginal hysterectomy (LAVH), and total vaginal hysterectomy (TVH). Discharges with the ICD-9 procedure code for "laparoscopic robotic-assisted procedure" (17.42 and 17.44) in combination with any of the hysterectomy codes were categorized as having undergone RALH. The laparoscopic retroperitoneal hysterectomy (LRH) approach was originally coded by the hospital as LAVH; however, after reading the operative reports, patients who underwent laparoscopic hysterectomy with retroperitoneal dissection (RPD) and uterine artery ligation at its vascular origin were identified as having undergone LRH. The LRH approach was given its own category, as it was consistently performed in the manner described below by a group of surgeons with a significant volume of cases large enough for meaningful comparison.

\section{LRH technique}

Conventional laparoscopy and robotic-assisted hysterectomy are performed with a wide variation in techniques and instrumentation that have been adequately described in the literature. We provide here the most important technical principles of the LRH technique as described in the operative reports. LRH is a variation of a total laparoscopic hysterectomy, with the distinguishing aspect of a standard retroperitoneal dissection (RPD), lateralization of the ureters, and ligation of the uterine artery at its origin from the anterior division of the internal iliac artery [14-16]. Abdominal entry is obtained by $5 \mathrm{~mm}$ direct entry trocar via the umbilicus and a $5 \mathrm{~mm}$ suprapubic port. A third port is placed in the right or left lower quadrant in more complex cases where additional assistance is required. With the ureter lateralized and in view, a defect is made in the posterior leaf of the broad ligament, which aids in isolating the infundibulopelvic ligament. The anterior leaf of the broad ligaments is then opened on each side to create a bladder flap that is carried through to the midline along the vesicouterine peritoneum. The anterior vaginal fornix is delineated using a simple sponge stick, and a colpotomy is created using the Harmonic scalpel. A Zumi manipulator is used for the manipulation of the uterus during the procedure. The uterus is then extracted vaginally, and if needed in cases of large specimen, by using extra-peritoneal vaginal debulking techniques. With LRH, the vaginal cuff is closed transvaginally, which, in our experience has led to faster operative times. Though retroperitoneal dissection, lateralization of the ureters, and early ligation of uterine arteries at the origin are techniques that can be performed in various ways with other approaches, the LRH technique was performed consistently as described above in the group of patients included in our analysis.

\section{Patient characteristics and operative outcomes}

Patient characteristics analyzed included age, race, weight, body mass index (BMI), surgical history, uterine weight, and uterine pathology. The Elixhauser Comorbidity Index was used to identify and record comorbid conditions that have been shown to potentially affect the operative outcomes [17], and those comorbidities were captured and recorded in each case. We also collected data on length of stay (LOS), which was counted as 0 if the patient was discharged before midnight on the same day of surgery. Surgical outcomes examined included the method of tissue extraction, estimated blood loss (EBL), skin-to-skin operative time (OT), intraoperative and postoperative complications, and rate of conversion from laparoscopic to abdominal hysterectomy. Minilaparotomy $(<5 \mathrm{~cm})$ was reported but not included as a "conversion" in the adjusted analysis due to the associated decreased blood loss, pain, tissue trauma, and LOS when compared to standard laparotomy [18]. EBL was obtained from the anesthesiology reports. Complications were defined as any undesirable, unintended, and direct outcome of surgery which affects the patient, which would not have occurred, had the surgery gone as well as could reasonably be hoped [19]. Complications were categorized as intraoperative when they occurred at the time of the procedure. Complications that presented within 60 days of the hysterectomy procedure and were treated at the hospital or the outpatient clinic were captured and categorized as postoperative complications.

\section{Costs}

While most comparative studies on hysterectomy use reimbursement amounts or hospital charge data [11, 20], our cost analysis was conducted from a healthcare system perspective, incorporating direct hospitalization costs associated with each procedure. We sought to improve the accuracy of cost estimation by including data on unit costs and resources utilized at the patient level $[21,22]$. Direct hospital costs included the following variables: room and board, physician reimbursement, operating room and anesthesia time, surgical equipment, and postoperative care (e.g., pharmacy, laboratory tests). To estimate physician reimbursement for each procedure, we used the 2013 OB/GYN Surgery Medicare reimbursement rates. The hospital's billing department provided the cost of room and board per day, preoperative costs of setup or time spent in the preoperative holding area, and the average per-minute costs for the operating room and anesthesia times. The latter fee schedule is based on a combination of nursing labor and fixed equipment for the operating room and is adjusted for every calendar year. Detailed equipment costs were obtained from the hospital's purchasing department. Costs of all disposable equipment (e.g., drapes, sealers, scalpel 
blades, trocars, forceps) required for each surgical procedure were included in the cost analysis (Appendix). Reusable instruments were assumed to have no cost as no additional investment was needed. Similarly, the acquisition and amortization of the da Vinci robot were not factored in, as the initial capital investment and the annual maintenance costs of the robotic system were previously funded by the hospital. Although we included a flat fee for preoperative times, it is important to note that this does not take into account personnel costs related to robot setup and disassembly and the impact on room turnover time [20]. The estimate of postoperative care, which includes pharmacy and laboratory tests, was also provided by the hospital's billing department. A standard cost to charge ratio of .6 was applied to the postoperative care estimate, as only charge data was provided by the hospital. Average costs were computed at the procedure level as well as at the patient level in order to provide more accurate estimates of the average, median, and range of costs (operating room + LOS + equipment + surgeon fee + postoperative care).

\section{Patient satisfaction}

The third arm of our value-based analysis drew on survey results of patient satisfaction within the context of an assessment of the patient decision-making process in choosing the route of hysterectomy. Patient satisfaction is multi-layered and contextual, and is conceptualized largely based on the congruence of expectations and subjective perception of the personalized service, care, and the final surgical outcome.

An IRB-approved survey was mailed to the same study population $(N=2689)$ in June 2015 . A unique survey identifier allowed us to match surveys with patient medical charts in order to validate the procedure route. The questions were derived from the existing standardized instruments [23, 24]. We use the following questions as a proxy for "satisfaction" with the overall surgical experience:

1. How satisfied are you with the surgery?
(a) Very satisfied
(b) Somewhat satisfied
(c) Somewhat dissatisfied
(d) Very dissatisfied
(e) I do not know

2. Compared to before the surgery, how would you rate your gynecological health now?
(a) Better now
(b) About the same
(c) Worse now
(d) I do not know

3. Would you recommend the same type of hysterectomy?
(a) Yes
(b) No
(c) I do not know

For each question, we calculated the percentage of respondents that chose option "a" as our indicator of satisfaction. The cumulative average satisfaction score was used in the value equation described below.

\section{Assessing value}

The need to use quality and cost to assess value is well-established, but there is no clear consensus on how to operationalize these two fundamental parameters [7]. For the purpose of this study, we defined quality as short-term operative outcomes plus overall patient satisfaction with the hysterectomy procedure:

$$
\text { value }=\text { quality } \div \text { direct hospital costs }
$$

where

$$
\text { quality }=\text { operative outcomes }+ \text { patient satisfaction }
$$

and

$$
\begin{aligned}
\text { operative outcomes }= & \text { operating time }+\mathrm{EBL} \\
& + \text { complications } \\
& + \text { conversion rate }
\end{aligned}
$$

Based on Ryan and Tompkins' "unconditional model," quality was quantified by creating a summary composite score of the unadjusted average OT, EBL, complication and conversion rates, and patient satisfaction [4]. Scores were calculated using quartiles. Operative outcome scores were assigned a weight of $75 \%$, and patient satisfaction score was assigned a weight of $25 \%$ of the quality score, which mirrors the Center for Medicare and Medicaid Services' pay for performance model [3].

\section{Statistical analysis}

Patients' demographic and clinical characteristics measured on a nominal or ordinal scale were summarized as counts and percentages, and compared across hysterectomy procedures by using Pearson's chi-squared tests; variables measured in the interval scale were summarized as means and standard deviations and compared across hysterectomy procedures by using a one-factor analysis of variance (ANOVA). Operative outcomes were compared unadjusted and also adjusted for age, race, number of previous abdominal surgeries, uterine size, BMI, number of additional procedures, number of comorbidities, and weight. Surgeon's experience was also controlled for by including the actual number of hysterectomies performed during the study period as a covariate in the models. 
For unadjusted comparisons of continuous clinical outcome (EBL, LOS), one-factor ANOVA was used to test the overall mean differences, followed by all-pairs Tukey-Kramer post hoc comparisons to identify means that were significantly different from each other. Unadjusted comparisons of dichotomous clinical outcomes (intraoperative complications, postoperative complications) were performed using logistic regression without covariates. Similarly, conversion (none, minilaparotomy, laparotomy) was analyzed as a polygamous outcome with multinomial logistic regression. We used post hoc Bonferroni-adjusted comparisons of predicted probabilities to identify groups that differed significantly while maintaining the overall significance probability at $5 \%$.

For adjusted analyses, linear regression was used to model EBL, logistic regression was used to model complications and conversions, and negative binomial regression was used to model LOS. Because of the concerns about non-normality of the dependent variable, we used median regression to model EBL and surgery time. We used adjusted (marginal) medians, proportions or counts, and corresponding delta method standard errors computed from the fitted models to perform Bonferroni-adjusted post hoc comparisons to identify groups that differed significantly while maintaining the overall significance probability at $5 \%$.
All statistical analyses were conducted with SPSS 21 (IBM Corp., Armonk, NY). All statistical tests were two-tailed at the $p<.05$ level.

\section{Results}

We reviewed the medical records of 2689 patients who underwent minimally invasive hysterectomy, with the following patient distribution from highest to lowest: $\mathrm{LRH}=$ $756, \mathrm{RALH}=576, \mathrm{LSH}=471, \mathrm{LAVH}=404, \mathrm{TLH}=361$, and $\mathrm{TVH}=121$. Age, weight, and BMI were equally distributed for each of the surgical groups (Table 1). Indications for surgery across procedures were similar, except for pelvic prolapse which was more highly represented in the TVH group. Indications for all procedures, in order of prevalence, greatest to least, included leiomyoma, abnormal menstruation, endometriosis, pelvic prolapse, endometrial hyperplasia, postmenopausal bleeding, benign ovarian cyst or neoplasm, cervical dysplasia, uterine polyp, and prophylactic organ removal. The percentage of patients with comorbidities and prior abdominal surgeries were also similar across procedures. The most significant difference in demographic characteristics was race; there was a higher percentage of African-American women in the LSH group. The average uterine specimen weight from highest to lowest was as follows: LSH $(410.4 \mathrm{~g}), \mathrm{LRH}$ (328.9 g), TLH (283.1 g), RALH (276.8 g), LAVH (273.6 g), and TVH (153.2 g) (Table 1).

Table 1 Patient characteristics

\begin{tabular}{|c|c|c|c|c|c|c|c|}
\hline Variable, mean (SD) ${ }^{a}$ & $\mathrm{LRH}$ & LSH & RALH & $\mathrm{TLH}$ & LAVH & $\mathrm{TVH}$ & $p$ value \\
\hline Age, years $^{b}$ & $47.7(9.5)$ & $45.5(5.7)$ & $48.9(8.8)$ & $47.8(9.0)$ & $48.4(9.1)$ & 49 (11.8) & $<0.0001$ \\
\hline Uterine size, $\mathrm{gm}^{\mathrm{b}}$ & $328.9(343.1)$ & 410.4 (461.6) & $276.8(266.3)$ & $283.1(292.2)$ & $273.6(301.8)$ & $153.2(101.6)$ & $<0.0001$ \\
\hline Weight, $\mathrm{kg}^{\mathrm{b}}$ & $79.5(21.1)$ & $84.8(21.4)$ & $86(26.4)$ & 78.4 (20.4) & $80.4(21.5)$ & $79.3(22.0)$ & $<0.0001$ \\
\hline $\mathrm{BMI}, \mathrm{k} / \mathrm{m}^{2 \mathrm{~b}}$ & $29.6(7.3)$ & $31.6(7.4)$ & $32(9.6)$ & $29.5(7.1)$ & $30.3(8.4)$ & $29.9(7.5)$ & $<0.0001$ \\
\hline \multicolumn{8}{|l|}{ Race $(\%)^{c}$} \\
\hline Black & 45 & 71 & 41 & 39.1 & 44.8 & 40 & \\
\hline White & 46.8 & 20 & 50 & 42.4 & 42.3 & 42 & \\
\hline Other & 6.7 & 8 & 9 & 17.5 & 11.9 & 18 & $<0.0001$ \\
\hline \multicolumn{8}{|l|}{ Prev abdominal } \\
\hline \multicolumn{8}{|l|}{ Surgeries $(\%)^{c}$} \\
\hline None & 33.1 & 29.3 & 30.6 & 34.3 & 31.5 & 40.5 & \\
\hline 1 & 33.6 & 34.4 & 30.9 & 35.2 & 38.7 & 37.2 & \\
\hline 2 & 20 & 23.6 & 21.9 & 19.9 & 19.1 & 17.4 & \\
\hline$>2$ & 13.4 & 12.7 & 16.7 & 10.5 & 10.7 & 5 & $<0.0001$ \\
\hline \multicolumn{8}{|c|}{ Comorbid conditions $(\%)^{c}$} \\
\hline None & 44.4 & 41.2 & 34 & 46 & 42.6 & 45.5 & \\
\hline 1 & 32.7 & 35.7 & 33.2 & 29.4 & 32.2 & 28.1 & \\
\hline$\geq 2$ & 22.9 & 23.1 & 32.8 & 24.7 & 25.2 & 26.4 & $<0.0001$ \\
\hline Total cases & 756 & 471 & 576 & 361 & 404 & 121 & \\
\hline
\end{tabular}

$L R H$ laparoscopic retroperitoneal hysterectomy, $L S H$ laparoscopic supracervical hysterectomy, $R A L H$ robotically assisted laparoscopic hysterectomy, $T L H$ total laparoscopic hysterectomy, $L A V H$ laparoscopically assisted vaginal hysterectomy, $T V H$ total vaginal hysterectomy, $B M I$ body mass index, SD standard deviation anless otherwise indicated, values are given as mean (standard deviation)

${ }^{\mathrm{b}}$ ANOVA, analysis of variance, $p<0.001$

${ }^{c}$ Chi-squared test $p<0.001$ 
The adnexa was removed in $4.8 \%$ of TVH cases, which was significantly less when compared to LRH $=12.7 \%$, $\mathrm{LSH}=16.9 \%, \mathrm{RALH}=18.8 \%, \mathrm{LAVH}=19.7 \%$, and $\mathrm{TLH}$ $=23.1 \%$.

All of the LRH cases and the majority of RALH cases (73.3\%) were performed by high-volume surgeons (high volume is defined here as $>30$ hysterectomies per year). The majority of LSH and TVH cases were performed by low-volume surgeons (54.6\% and $89.8 \%$, respectively). The percentage of low-, intermediate-, and high-volume surgeons was more equally distributed amongst the TLH and LAVH cases (Table 2).

Analysis of the operative outcomes across procedures showed statistically significant differences in EBL, LOS, OT, complications, and rate of conversion to abdominal hysterectomy. Operative outcomes were compared across hysterectomy procedures with and without adjustments for demographics (age, race, BMI), case complexity factors (number of previous surgeries, uterine size, number of additional procedures, number of comorbidities, weight), and surgeon volume. Unadjusted results are summarized in Table 3 . Adjusted results are summarized in Table 4 and are discussed below.

LRH had the lowest mean EBL $(100.1 \mathrm{~mL} ; p<.0001)$, which was statistically significant when compared with LAVH and TVH. There were two intraoperative blood transfusions (1, LAVH; 1, LRH) and two postoperative transfusions (1, RALH; 1, LAVH).

LRH had the shortest and LSH the longest operative times compared with all other groups $(p<.0001)$. The mean OT from shortest to longest were as follows: LRH (71.2 min), TVH (93.1 min), RALH (99.6 min), TLH (110.9 min), LAVH (117.0 min), and LSH (119.9 min).

$\mathrm{TVH}$ had the highest rate of intraoperative complications (9.6\%); LRH had the lowest $(2.1 \% ; p=.0033)$, which was statistically significant compared to RALH, TLH, LAVH, and TVH $(p<.0001)$. The rate of postoperative complications was highest in the RALH group (11.4\%); TVH had the lowest (1.8\%). The overall incidence of vaginal cuff dehiscence within the 60-day postoperative period was rare in our analysis at $0.45 \%$. The dehiscence

Table 2 Surgeon volume

\begin{tabular}{llll}
\hline & Low volume (\%) & Intermediate volume (\%) & High volume (\%) \\
\hline LRH & 0 & 0 & 100 \\
LSH & 54.60 & 31.10 & 12.50 \\
RALH & 8.90 & 17.80 & 73.30 \\
TLH & 37.10 & 24.10 & 38.80 \\
LAVH & 33.30 & 28.50 & 38.20 \\
TVH & 89.80 & 6.10 & 4.10
\end{tabular}

LRH laparoscopic retroperitoneal hysterectomy, $L S H$ laparoscopic supracervical hysterectomy, $R A L H$ robotically assisted laparoscopic hysterectomy, $T L H$ total laparoscopic hysterectomy, LAVH laparoscopically assisted vaginal hysterectomy, $T V H$ total vaginal hysterectomy percentage per procedure (lowest to highest) was as follows: TVH $0.0 \%$, LSH $0.0 \%$, LAVH $0.25 \%$, LRH $0.53 \%$, RALH $0.52 \%$, and TLH $1.1 \%$. The most frequently occurring intra- and postoperative complications are listed in Table 5. Conversion to abdominal hysterectomy occurred in 14 cases: 9 cases in the TLH group, 4 in LSH, and 1 in RALH. There were no conversions to abdominal hysterectomy in LAVH, LRH, or TVH.

\section{Costs}

There were significant cost differences across procedures, which reflected similar cost comparisons found in the literature $[11,25,26]$. Examining the average of the entire cohort, LRH was the least expensive with an average cost of $\$ 4061$, ranging from $\$ 2782$ to $\$ 10,687$ (Fig. 1). This is approximately half the cost of LSH, RALH, TLH, and LAVH. The lower costs associated with LRH were attributed to the use of reusable instruments, shorter OT, and shorter LOS. TVH had the second lowest average cost at $\$ 6416$. RALH had the highest cost with an average of $\$ 9354$, ranging from $\$ 6019$ to $\$ 19,815$, which was driven primarily by OT, LOS, and disposable instruments (Table 6).

\section{Patient satisfaction}

The response rate of the survey was $12 \%(N=322)$, which falls within the expected average $10-15 \%$ rate of response for external mail surveys with no monetary incentive [27]. The average mean patient satisfaction scores ranged from 79.1 to 87.2. LRH had the highest combined patient satisfaction score, followed by RALH, while TLH, LAVH, TVH, and LSH averaged similar scores as shown in Table 7.

\section{Value}

We applied our value formula (value = quality/costs) to each hysterectomy procedure approach, where a summary composite score of the unadjusted average OT, EBL, complication and conversion rates, and patient satisfaction were calculated via equally weighted quartiles [28].

LRH, TVH, and LAVH yielded the highest value scores, while LSH, TLH, and RALH yielded the lowest scores (Table 8). There was little meaningful difference between the lower scores.

\section{Discussion}

Healthcare spending has been growing as a share of national income in the USA for decades-from $10 \%$ in 1985 to $17 \%$ in 2010 - and it is projected to keep rising to almost $25 \%$ in 2037 [29]. The costs continue to escalate in large part because of medical technology. Healthcare economists estimate that $40-50 \%$ of annual cost increases can be traced to new technologies, such as the 
Table 3 Unadjusted analysis of operative outcomes

\begin{tabular}{llllllll}
\hline Variable, mean (SD) & LRH & LSH & RALH & TLH & LAVH & TVH & $p$ value \\
\hline Number of ports & $2.2(0.5)$ & $3.2(0.5)^{*}$ & $4.7(0.6)^{*}$ & $3.4(0.6)^{*}$ & $3.2(0.5)^{*}$ & N/A & $<0.0001$ \\
EBL $(\mathrm{mL})$ & $129.3(142.1)$ & $197.5(263.2)^{*}$ & $118.6(133.8)$ & $165.8(177.3)^{*}$ & $200.7(210.4)^{*}$ & $244.7(240.8)^{*}$ & $<0.0001$ \\
LOS (days) & $0.2(0.5)$ & $0.6(0.7)^{*}$ & $1.1(1.0)^{*}$ & $0.8(0.8)^{*}$ & $0.8(0.8)^{*}$ & $1.2(0.6)^{*}$ & $<0.0001$ \\
OT (min) & $61.8(31.0)$ & $140.9(53.5)^{*}$ & $111.7(52.0)^{*}$ & $126.8(70.5)^{*}$ & $133.3(63.7)^{*}$ & $113.6(64.3)^{*}$ & $<0.0001$ \\
RPD, \% (N) & $99.5(752)$ & $12.7(60)^{*}$ & $35.3(203)^{*}$ & $18.3(66)^{*}$ & $23.9(97)^{*}$ & $\mathrm{~N} / \mathrm{A}$ & $<0.0001$ \\
Prob IntraOp Comp, \% (N) & $2.2(17)$ & $4.9(23)$ & $5.4(31)^{*}$ & $5.5(20)^{*}$ & $6.9(28)^{*}$ & $6.6(8)^{*}$ & 0.005 \\
Prob PostOp Comp, \% (N) & $3.2(24)$ & $6.2(29)$ & $10.8(62)^{*}$ & $5.0(18)$ & $8.4(34)^{*}$ & $2.0(2)$ & $<0.0001$ \\
Prob Convert Open, \% (N) & $1 .(0)$ & $0.8(4)$ & $0.2(1)$ & $2.5(9)^{*}$ & $0.0(0)$ & $1 .(0)$ & $\mathrm{N} / \mathrm{A}$ \\
Total cases & 756 & 471 & 576 & 361 & 404 & 121 & \\
\hline
\end{tabular}

$L R H$ laparoscopic retroperitoneal hysterectomy, $L S H$ laparoscopic supracervical hysterectomy, $R A L H$ robotically assisted laparoscopic hysterectomy, $T L H$ total laparoscopic hysterectomy, $L A V H$ laparoscopically assisted vaginal hysterectomy, $T V H$ total vaginal hysterectomy, $R P D$ retroperitoneal dissection, $E B L$ estimated blood loss, LOS length of stay, OT operative time, Prob probability, IntraOp intraoperative, PostOp postoperative, Comp complications, Prob Convert Open probability of conversion to laparotomy

*Statistical significance; Bonferroni adjusted $p<0.05$ comparing each procedure to LRH

${ }^{a}$ Unless otherwise indicated, values are given as mean (standard deviation)

da Vinci robot [30]. A corrective shift toward a valuebased model is needed to move the healthcare system in the direction of greater sustainability, offering benefits to the provider, payor, and the patient [31].

An approach similar to the summary composite score used by US News and World Report to rank hospitals should be considered to compare surgical modalities. Past studies on hysterectomy have considered operative outcomes and cost separately, but in today's value-driven healthcare marketplace, it is important to evaluate surgical outcomes and patient satisfaction relative to cost. While the application of the value equation introduced in this study is novel and untested, it is an attempt to refocus research efforts on the concept of value in surgical interventions.

It is noteworthy that while TVH had the highest rate of intraoperative complications of all procedures and was on the lower end of patient satisfaction scores, it had the lowest postoperative complication rate and lowest average cost, yielding a higher value score than TLH, RALH, LAVH, and LSH. Of note, the adjusted intraoperative complication rate for TVH is high because it was performed on small uterine sizes and with a small number of additional procedures. The regression model

Table 4 Adjusted analysis of operative outcomes

\begin{tabular}{|c|c|c|c|c|c|c|c|}
\hline $\begin{array}{l}\text { Variable, adj. median (95\% } \\
\mathrm{Cl}^{\mathrm{a}}\end{array}$ & $\mathrm{LRH}$ & LSH & RALH & $\mathrm{TLH}$ & LAVH & $\mathrm{TVH}$ & $p$ value \\
\hline $\begin{array}{l}\text { Number of ports-adj. } \\
\text { counts }\end{array}$ & $2.1(2.0,2.2)$ & $3.3(3.1,3.5)^{*}$ & $4.7(4.5,4.9)^{*}$ & $3.5(3.3,3.7)^{*}$ & $3.3(3.1,3.5)^{*}$ & N/A & $\begin{array}{l}< \\
0.0001\end{array}$ \\
\hline $\mathrm{EBL}(\mathrm{mL})$ & $\begin{array}{l}100.1(90.3 \\
109.9)\end{array}$ & $\begin{array}{l}116.6(106.1 \\
127.90)\end{array}$ & $\begin{array}{l}100.9(92.1 \\
109.7)\end{array}$ & $\begin{array}{l}114.5(103.2, \\
125.7)\end{array}$ & $\begin{array}{l}135.9(125.1 \\
146.7)^{*}\end{array}$ & $\begin{array}{l}188.9(169.2, \\
208.6)^{*}\end{array}$ & $\begin{array}{l}< \\
0.0001\end{array}$ \\
\hline LOS (days) & $0.2(0.2,0.2)$ & $0.5(0.5,0.6)^{*}$ & $1.1(1.0,1.2)^{*}$ & $0.8(0.7,0.9)^{*}$ & $0.8(0.7,0.9)^{*}$ & $1.2(1.0,1.4)^{*}$ & $\begin{array}{l}< \\
0.0001\end{array}$ \\
\hline OT (min) & $71.2(66.9,75.5)$ & $\begin{array}{l}119.9(115.3 \\
124.6)^{*}\end{array}$ & $\begin{array}{l}99.6(95.7 \\
103.6)^{*}\end{array}$ & $\begin{array}{l}110.9(105.8 \\
115.9)^{*}\end{array}$ & $\begin{array}{l}117.0(112.1 \\
121.8)^{*}\end{array}$ & $93.1(84.3,101.9)^{*}$ & $\begin{array}{l}< \\
0.0001\end{array}$ \\
\hline Prob. IntraOp Comp, \% & $2.1(1.0,3.2)$ & $5.1(2.9,7.3)$ & $5.6(3.7,7.6)^{*}$ & $5.8(3.3,8.3)^{*}$ & $7.1(4.4,9.8)^{*}$ & $9.6(3.1,16.0)^{*}$ & 0.0033 \\
\hline Prob. PostOp Comp, \% & $3.6(1.9,5.2)$ & $4.9(3.0,6.9)$ & $11.4(8.7,14.0)^{*}$ & $5.1(2.8,7.5)$ & $8.4(5.6,11.3)^{*}$ & $1.8(0.0,4.3)$ & $\begin{array}{l}< \\
0.0001\end{array}$ \\
\hline Prob. Convert Open, \% & $0.0(0.0,0,0)$ & $1.0(0.0,1.9)$ & $0.4(0.0,1.2)$ & $4.8(1.5,8.2)$ & $0.0(0.0,0.0)$ & $0.0(0.0,0.0)$ & N/A \\
\hline Total cases & 756 & 471 & 576 & 361 & 404 & 121 & \\
\hline
\end{tabular}

Adjusted for age, race, number of previous abdominal surgeries, BMI, number of comorbidities, weight, uterine size, number of additional procedures, and surgeon's volume

$L R H$ laparoscopic retroperitoneal hysterectomy, $L S H$ laparoscopic supracervical hysterectomy, $R A L H$ robotically assisted laparoscopic hysterectomy, $T L H$ total laparoscopic hysterectomy, LAVH laparoscopically assisted vaginal hysterectomy, TVH total vaginal hysterectomy, EBL estimated blood loss, LOS length of stay, OT operative time, IntraOp intraoperative, PostOp postoperative, Comp complications, Prob Convert Open probability of conversion to laparotomy *Statistical significance in post hoc analysis comparing each surgical procedure to LRH. Significance probability corrected to maintain the family-wise error rate at $a=0.05$

${ }^{a}$ Unless otherwise indicated, values are given as adjusted medians $(95 \% \mathrm{Cl})$ 


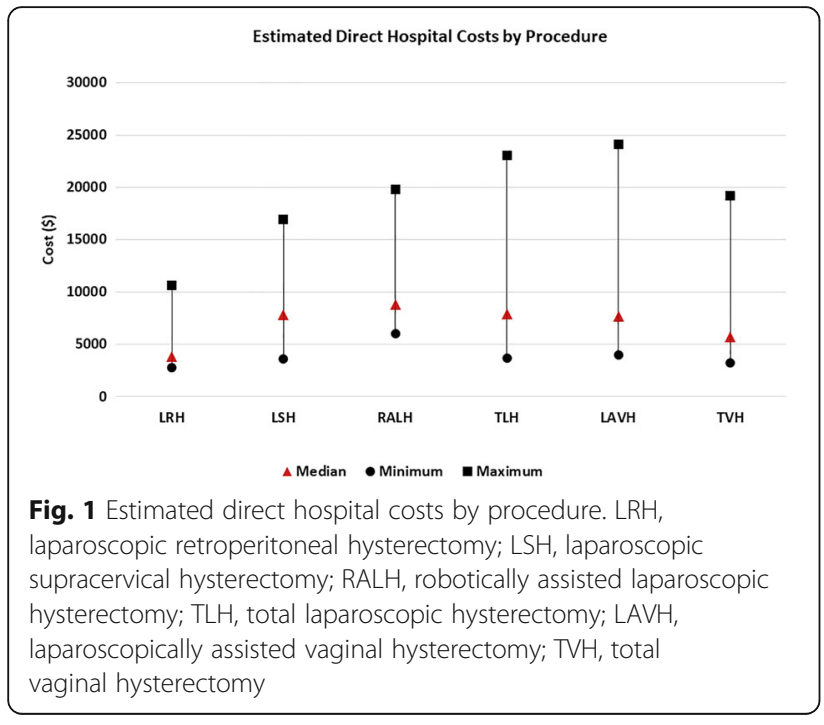

estimated the complication rate if the uterine size and number of additional procedures were equal to the average observed in the data. If the uterine size and number of additional procedures were removed from the model, the adjusted estimate for intraoperative complication would change from 9.6 to $6.8 \%$ (CI 2.0, 11.7).

RALH rated high in patient satisfaction, but had the highest rate of postoperative complications and was the most expensive procedure, yielding a lower value score. Robotic hysterectomy offers certain advantages to the surgeon compared to conventional laparoscopic surgery, such as better ergonomics, improved dexterity, and enhanced visualization [32]. This technology has transformed the field of gynecological surgery by enabling a greater number of surgeons to offer minimally invasive procedures, thereby greatly reducing the rate of open abdominal procedures. However, despite its surge in popularity since the introduction of robotic surgery in 2005, it has not shown improved surgical outcomes, and as this and other studies have shown, its costs are significantly greater than other laparoscopic procedures [32, 33]. The financial burden may not be justified when other hysterectomy techniques may equal or surpass the operative outcomes of robotics at a much lower cost.

LRH yielded the highest value score, driven primarily by the low intraoperative complication rate, high patient satisfaction scores, and low costs. The low rate of intraoperative complications, which contributed to a shorter operative time and length of stay, is due in part to surgeon experience but may also be attributed to the standard retroperitoneal dissection, which allows for full visualization and lateralization of the ureters, and early control of the blood supply by ligation of the uterine arteries at the vascular origin.

Table 5 Most common complications

\begin{tabular}{|c|c|c|c|c|c|c|c|}
\hline Complications $^{\mathrm{a}, \mathrm{b}}(\%)$ & $\mathrm{LRH}$ & LSH & RALH & TLH & LAVH & $\mathrm{TVH}$ & Total \\
\hline \multicolumn{8}{|l|}{ Intraoperative } \\
\hline $\mathrm{EBL}>1000 \mathrm{~mL}$ & $1(.13)$ & $7(1.5)$ & $2(.34)$ & $2(.55)$ & $6(1.5)$ & $2(1.65)$ & $20(.74)$ \\
\hline Cystotomy & $2(.26)$ & $3(.63)$ & $3(.52)$ & $2(.55)$ & $4(.99)$ & $3(2.48)$ & $17(.63)$ \\
\hline Ureteral injury & $2(.26)$ & $0(0.0)$ & $5(.86)$ & $0(0.0)$ & $3(.74)$ & $1(.83)$ & $11(.41)$ \\
\hline Enterotomy & $0(0.0)$ & $0(0.0)$ & $2(.34)$ & $2(.55)$ & $5(1.24)$ & $1(.83)$ & $10(.37)$ \\
\hline Urethral injury & $0(0.0)$ & $1(.21)$ & $1(.17)$ & $0(0.0)$ & $0(0.0)$ & $0(0.0)$ & $2(.074)$ \\
\hline Blood transfusion & $1(.13)$ & $0(0.0)$ & $0(0.0)$ & $0(0.0)$ & $1(.25)$ & $0(0.0)$ & $2(.074)$ \\
\hline Iliac vein injury & $1(.13)$ & $0(0.0)$ & $0(0.0)$ & $1(.28)$ & $0(0.0)$ & $0(0.0)$ & $2(.074)$ \\
\hline Rectal injury & $1(.13)$ & $0(0.0)$ & $1(.17)$ & $0(0.0)$ & $0(0.0)$ & $0(0.0)$ & $2(.074)$ \\
\hline \multicolumn{8}{|l|}{ Postoperative } \\
\hline Vaginal cuff dehiscence & $4(.53)$ & $0(0.0)$ & $3(.52)$ & $4(1.1)$ & $1(.25)$ & $0(0.0)$ & $12(.45)$ \\
\hline Pelvic infection/abcess & $4(.53)$ & $4(.84)$ & $19(3.2)$ & $1(.28)$ & $2(.50)$ & $0(0.0)$ & $30(1.1)$ \\
\hline Pulmonary embolus & $1(.13)$ & $1(.21)$ & $0(0.0)$ & $0(0.0)$ & $1(.25)$ & $0(0.0)$ & $3(.11)$ \\
\hline Bacteremia & $0(0.0)$ & $0(0.0)$ & $2(.34)$ & $0(0.0)$ & $0(0.0)$ & $0(0.0)$ & $2(.074)$ \\
\hline DVT & $1(.13)$ & $0(0.0)$ & $0(0.0)$ & $1(.28)$ & $0(0.0)$ & $0(0.0)$ & $2(.074)$ \\
\hline Cuff cellulitis & $0(0.0)$ & $0(0.0)$ & $0(0.0)$ & $0(0.0)$ & $2(.50)$ & $0(0.0)$ & $2(.074)$ \\
\hline Blood transfusion & $0(0.0)$ & $0(0.0)$ & $1(.17)$ & $0(0.0)$ & $1(.25)$ & $0(0.0)$ & $2(.074)$ \\
\hline Total cases & 756 & 471 & 576 & 361 & 404 & 121 & 2689 \\
\hline
\end{tabular}

LRH laparoscopic retroperitoneal hysterectomy, LSH laparoscopic supracervical hysterectomy, $R A L H$ robotically assisted laparoscopic hysterectomy, $T L H$ total laparoscopic hysterectomy, LAVH laparoscopically assisted vaginal hysterectomy, $T V H$ total vaginal hysterectomy, EBL estimated blood loss, DVT deep vein thrombosis

${ }^{a}$ Only the most common complications reported here

${ }^{\mathrm{b}}$ Includes multiple complications as dictated in operative notes, but counted as one event in statistical analysis to avoid double counting 
Table 6 Hospital costs

\begin{tabular}{|c|c|c|c|c|c|c|}
\hline & $\mathrm{LRH}$ & LSH & RALH & TLH & LAVH & $\mathrm{TVH}$ \\
\hline LOS at $\$ 1043 /$ day & 208.60 & 730.10 & 1147.30 & 834.40 & 730.10 & 1251.60 \\
\hline Equipment ${ }^{1,2}$ & 671.00 & 1915.00 & 2972.00 & 1915.00 & 1915.00 & 304.00 \\
\hline OR time at $29.22 / \mathrm{min}^{1}$ & 1577.90 & 4207.70 & 3272.60 & 3710.90 & 3652.50 & 3155.80 \\
\hline Anesth at $1.13 / \mathrm{min}^{1}$ & 61.00 & 162.70 & 126.60 & 143.50 & 141.30 & 122.00 \\
\hline Surgeon fee ${ }^{3}$ & 1181.00 & 1018.00 & 1181.00 & 1204.00 & 1181.00 & 859.00 \\
\hline Postop care (pharmacy + labs) ${ }^{1}$ & 353.00 & 331.00 & 591.00 & 530.00 & 353.00 & 505.00 \\
\hline Total Est. hospital costs & 4052.50 & 8364.50 & 9290.50 & 8337.80 & 7972.90 & 6197.40 \\
\hline \multicolumn{7}{|l|}{ Entire cohort at patient level } \\
\hline Mean (SD) & 4060.5 (931.9) & $8227.1(1898.9)$ & 9353.8 (2103.9) & $8372.1(2531.8)$ & 8054.5 (2352.6) & $6415.8(2331.1)$ \\
\hline Median & 3813.6 & 7888.3 & 8852.3 & 7898 & 7678.8 & 5776.3 \\
\hline Range & $2781.7,10,686.7$ & $3597.8,16,932.6$ & $6018.7,19,814.6$ & $3679.3,23,064.8$ & $4025.7,24,161.8$ & $3257.3,19,194.0$ \\
\hline Total cases & 756 & 471 & 576 & 361 & 404 & 121 \\
\hline
\end{tabular}

$L R H$ laparoscopic retroperitoneal hysterectomy, $L S H$ laparoscopic supracervical hysterectomy, $R A L H$ robotically assisted laparoscopic hysterectomy, $T L H$ total laparoscopic hysterectomy, LAVH laparoscopically assisted vaginal hysterectomy, TVH total vaginal hysterectomy, LOS length of stay, OR operating room, Anesth anesthesia, Postop postoperative, Labs laboratory, Est estimate

${ }^{1}$ Based on the information provided by the hospital billing department

${ }^{2}$ Equipment costs provided by the hospital purchase department; equipment used in each procedure provided by senior OR nurse

${ }^{3}$ Based on 2014 OB/GYN Surgery Medicare reimbursement rates

The concept of value should also be considered within the context of surgeon training and expertise. Studies have shown that surgeon volume and experience are important factors influencing the chosen route of hysterectomy, operative outcomes, and cost [34, 35]. High-volume gynecologic surgeons have been shown to improve multiple patient outcomes including decreased blood loss, operative time, intraoperative and postoperative complications, ICU admissions, transfusions, hospital readmissions, and length of hospital admissions. A linear relationship has also been demonstrated between surgeon volume and cost, with a decrease in costs as surgeon volume increases [36]. The techniques inherent to LRH require the skills of advanced laparoscopy and in this study were performed by high-volume surgeons.

It is also significant to note that only 5 out of 121 TVH cases in our study were performed by high-volume surgeons. TVH has traditionally been considered the gold standard of minimally invasive hysterectomy and is the recommended route by the American College of Obstetricians and Gynecologists whenever feasible, while laparoscopic hysterectomy is the recommended alternative to abdominal hysterectomy when TVH is not indicated or feasible [37]. While TVH ranked highly in our analysis in terms of value, this approach offers limited surgical views and access to the pelvis and may be challenging to treat pathology such as grossly enlarged leiomyomatous uteri or endometriosis. Additionally, with the emerging evidence that epithelial ovarian malignancies arise in the fallopian tubes and spread to the ovaries, there is a recommended clinical practice shift toward prophylactic salpingectomy at the time of hysterectomy [38]. While it is feasible to remove the fallopian tubes during vaginal hysterectomy, studies have acknowledged the challenges and limitations associated with this approach and have reported a higher success rate of adnexal removal amongst experienced vaginal surgeons [39]. However, a 2011 survey found that only $28 \%$ of

Table 7 Patient satisfaction scores (\%)

\begin{tabular}{|c|c|c|c|c|c|c|c|}
\hline & $\mathrm{LRH}$ & LSH & RALH & TLH & LAVH & $\mathrm{TVH}$ & Total \\
\hline Overall very satisfied with surgery? ${ }^{a}$ & 87.5 & 73.1 & 85.7 & 86.7 & 81.8 & 73.4 & \\
\hline Compared to before surgery, is GYN health better now? ${ }^{b}$ & 94.8 & 86.3 & 92.7 & 93.1 & 90.9 & 92 & \\
\hline Would you recommend the same surgery? ${ }^{c}$ & 79.5 & 78 & 80.6 & 62.1 & 68.2 & 75 & \\
\hline Mean & 87.2 & 79.1 & 86.3 & 80.6 & 80.3 & 80.1 & \\
\hline Total cases (response rate within procedure) & $82(10.8)$ & $58(12.3)$ & $103(17.9)$ & $35(9.7)$ & $27(6.7)$ & $17(14.00)$ & $322(11.9)$ \\
\hline
\end{tabular}

LRH laparoscopic retroperitoneal hysterectomy, $L S H$ laparoscopic supracervical hysterectomy, RALH robotically assisted laparoscopic hysterectomy, $T L H$ total laparoscopic hysterectomy, $L A V H$ laparoscopically assisted vaginal hysterectomy, $T V H$ total vaginal hysterectomy

"Percentage of respondents indicating "very satisfied"

bPercentage of respondents indicating "much better"

"Percentage of respondents indicating "yes" 
graduating residents were "completely prepared" to perform a vaginal hysterectomy, compared to $58 \%$ for abdominal hysterectomy, 22\% for laparoscopic, and 3\% for robotic hysterectomy [40]. Greater emphasis in residency and fellowship training programs should be given to hysterectomy approaches and techniques that offer better value with proven surgical outcomes and high levels of patient satisfaction at lower costs.

\section{Limitations}

The current study has several limitations. Its retrospective nature is limited by inherent selection bias. Although tests were conducted to ensure inter- and intra-rater reliability amongst data abstractors, the availability and accuracy of the medical records, as well as transcription errors, remain intrinsic limitations.

All hospital data on reoperation and readmittance within 60 days were collected; however, the total number of postoperative complications may be underreported, as patients with adverse events may have been seen in their physician's office or at a different hospital. In the same vein, we were not able to capture the costs of readmissions or reoperations; however, these occurrences are reflected in the complication rates.

Another limitation is that the different hysterectomy approaches were not performed by the same surgeons. The three surgeons who performed all of the LRH procedures, and the three surgeons who performed $89 \%$ of RALH procedures, are skilled and experienced laparoscopists who perform a high volume of cases. The three high-volume robotics surgeons have each been performing robotics for over 10 years. Thus, there are limitations in making a direct comparison to other procedure types performed by different surgeons of varying experience, skill level, and practice patterns. As high-volume surgeons are associated with lower costs and better surgical outcomes [41], we attempted to make meaningful comparisons by controlling for surgeon volume in our regression models.

Further, length of stay was included in the cost analysis, but it should be noted that LOS is often a protocol-driven outcome; it can be as much influenced by surgeon preference and the time of day of the procedure, as by the condition of the patient. However, when we omitted LOS from the cost calculation, the result was largely the same. It did not change the ranking of the value scores (Table 8).

Although the US Panel on Cost-Effectiveness in Health and Medicine recommends micro-costing as the preferred approach to cost estimation [42], one of the limitations of micro-costing is that it lacks external validity; there is a limit to the extent that one can generalize to other settings. It should also be noted that costs were calculated from a healthcare system perspective, incorporating direct hospitalization costs associated with each procedure. A more rigorous cost analysis should also incorporate the indirect costs to society, such as lost wages from work absence and differential productivity costs. Determining the costs of surgical procedures is never a straightforward calculation; however, we sought not to compute the conclusive costs of hysterectomy procedures, but rather to compare the relative cost differences between procedural routes.

Additionally, our operational definitions may not accurately capture the intended construct of "value." Past attempts to measure this concept required some type of weighting and threshold setting, which inevitably invite the stakeholder's preference. This remains one of the inherent limitations in measuring value; how it is defined dictates how measures are selected and weighted. Our value ratio was based on a summary composite score of short-term operative outcomes and patient satisfaction using quartiles. Because this specific approach has not been used in previous studies, there is no assessment of the reliability and validity of measures combining quality and cost. We did not find an alternative approach for combining quality and cost measures into quantifiable measures of value to evaluate surgical modalities.

Further, the patient perspective was based on a mail survey with high non-response bias. Although a $12 \%$ response rate is a typical response rate for an external mail survey with no monetary incentive, the low response

Table 8 Value scores

\begin{tabular}{lllll}
\hline & Composite quality score & Avg. hospital costs & Value score $^{\dagger}$ & Value ranking $^{\dagger}$ \\
\hline LRH & 11.5 & 4060.5 & 2.8 & 1 \\
LSH & 5.8 & 8227.1 & 0.7 & 4 \\
RALH & 6.0 & 9353.8 & 0.64 & 6 \\
TLH & 5.8 & 8372.1 & 0.69 & 5 \\
LAVH & 7.3 & 8054.5 & 0.9 & 3 \\
TVH & 8.0 & 6415.8 & 1.2 & 2 \\
\hline
\end{tabular}

LRH laparoscopic retroperitoneal hysterectomy, $L S H$ laparoscopic supracervical hysterectomy, $R A L H$ robotically assisted laparoscopic hysterectomy, $T L H$ total laparoscopic hysterectomy, $L A V H$ laparoscopically assisted vaginal hysterectomy, $T V H$ total vaginal hysterectomy

${ }^{\dagger}$ Value score $=$ composite quality score/avg. hospital costs; where: quality $=75 \%$ (OT + complications) $+25 \%$ (patient satisfaction) 
rate decreases the survey's internal validity, thereby limiting its representativeness [28]. Recall bias may also be a limitation, as the survey was administered between 1.5 and 4.5 years postoperatively. Additionally, patient satisfaction score was constructed with just three standardized questions regarding satisfaction. In order to build a more rigorous value model that includes the patient perspective, a prospective study is needed to investigate time to return to normal daily activity for each surgical procedure, as well as changes in the patient's quality of life using a validated quality of life instrument.

\section{Strengths}

Rather than examining the individual metrics alone, we used a novel formula of operative outcomes plus patient satisfaction divided by direct hospital costs in an attempt to operationalize and compare the value of the most common minimally invasive hysterectomy techniques.

One of the main strengths of the current study is the large sample size, with a wide array of surgeon experience, surgical techniques, patient demographics, and variations in pathology. To our knowledge, it is also the only study in this field employing a sequential, mixed method design, which included a retrospective chart review and a mail survey of the same patient population. This allowed us to integrate the patient perspective into our value framework.

Another strength is the inclusion of direct hospitalization costs rather than the oft-reported insurance reimbursement amounts or charge data $[11,20]$. Using a micro-costing approach from the healthcare system perspective, we included data on unit costs and resources utilized at the procedural and patient levels.

\section{Conclusion}

At a time of increasing demand for more fiscal responsibility in healthcare, the emphasis on more costly surgical interventions, such as robotics, should be considered carefully when less costly alternatives provide better value in terms of operative outcomes and patient satisfaction.

Rather than examining individual metrics alone, we used a value formula based on the Medicare pay-for-performance model to compare minimally invasive hysterectomy techniques. For healthcare stakeholders seeking to control costs without sacrificing quality, it is critical to operationalize the value of varying surgical techniques, including measures of surgeon experience, operative outcomes, costs, and patient satisfaction. While there are acknowledged limitations of the current study, our efforts serve to open a dialogue regarding a value-based model to evaluate surgical approaches, in an effort to push the healthcare system in the direction of greater sustainability.

\section{Appendix \\ Disposable equipment costs \\ Available upon request.}

\section{Abbreviations}

ACOG: American College of Obstetricians and Gynecologists; ANOVA: Analysis of variance; BMI: Body mass index; CMS: Centers for Medicare and Medicaid Services; CPT: Current Procedural Terminology; EBL: Estimated blood loss; ICD-9: International Classification of Diseases-Ninth revision; IRB: Institutional Review Board; LAVH: Laparoscopically assisted vaginal hysterectomy; LOS: Length of stay; LRH: Laparoscopic retroperitoneal hysterectomy; LSH: Laparoscopic supracervical hysterectomy; OT: Operative time; RALH: Robotically assisted laparoscopic hysterectomy;

RPD: Retroperitoneal dissection; TLH: Total laparoscopic hysterectomy; $\mathrm{TVH}$ : Total vaginal hysterectomy

\section{Acknowledgements}

We would like to thank June Kyrk, Kim Williams, and Patty Smith for their diligent data abstraction. Dr. Britton Chahine provided guidance in data collection and contributed to the first draft of this paper. We would also like to thank: Dr. Stella Blosser Lii for her critical review and recommendations regarding this publication; Kellie Washington and Sandra Staciu for overseeing the administration of the survey; Nilofar Kazi and Laila Kazi for their help in preparing the manuscript; Professor Dr. J. Jeekel for his critical review and recommendations regarding the first draft of this publication; as well as The Center for Innovative GYN Care for its continued commitment to excellence in research.

\section{Funding}

This study was funded by The Center for Innovative GYN Care. CIGC did not play a role in the design, collection, analysis, or interpretation of the data.

\section{Availability of data and materials}

All data has been summarized in the tables and figures in the manuscript. Raw data is available upon request from the corresponding author.

\section{Authors' contributions}

PM and ND contributed to the project development, study design, and editing. RB contributed to the project development and manuscript writing and editing. LV contributed to the study design, statistical analysis, and manuscript writing and editing. LH contributed to the data collection and management and manuscript writing and editing. All authors read and approved the final manuscript.

Ethics approval and consent to participate

This study was approved by the hospital's Institutional Review Board (Study Number 2017-01). For this type of study formal consent is not required.

\section{Consent for publication}

The data used in this study was patient clinical data, excluding unique identifiers. Therefore, given the retrospective nature of the study, informed consent was not required.

\section{Competing interests}

The authors declare they have no competing interests. Data abstractors were independent contractors with no vested interest in The Center for Innovative GYN Care. Dr. Natalya Danilyants and Dr. Paul MacKoul are both co-owners of The Center for Innovative GYN Care, and while they contributed to writing and editing of the manuscript, neither Dr. Danilyants nor Dr. MacKoul played a role in the collection, analysis, or interpretation of the data. Dr. Louise van der Does and Leah Haworth, BSN, RN are employed by The Center for Innovative GYN Care.

\section{Publisher's Note}

Springer Nature remains neutral with regard to jurisdictional claims in published maps and institutional affiliations. 


\section{Author details}

The Center for Innovative GYN Care, 3206 Tower Oaks Blvd., Suite 200, Rockville, MD 20852, USA. ${ }^{2}$ Holy Cross Hospital, 1500 Forest Glen Rd, Silver Spring, MD 20910, USA.

\section{Received: 2 February 2018 Accepted: 1 February 2019}

\section{Published online: 18 March 2019}

\section{References}

1. Health Services Research on Hysterectomy and Alternatives (1998) Agency for Healthcare Research and Quality. http://archive.ahrq.gov/research/hysterec.htm. Accessed 1 Nov 2016

2. Chow A, Mayer EK, Darzi AW, Athanasiou T (2009) Patient-reported outcome measures: the importance of patient satisfaction in surgery. Surgery 146(3): 435-443. https://doi.org/10.1016/j.surg.2009.03.019

3. CMS Publishes Data on Consumer Satisfaction for Hospitals (2015). http:// www.emds.com/cms-publishes-data-consumer-satisfaction-hospitals. Accessed 15 Aug 2017

4. Ryan AM, Tompkins CP (2014) Efficiency and value in healthcare: linking cost and quality measures. National Quality Forum. http:// www.qualityforum.org/Publications/2014/11/Efficiency_and_Value_in_ Healthcare_Linking_Cost_and_Quality_Measures_Paper.aspx. Accessed 10 July 2017

5. Timbie JW, Normand SL (2008) A comparison of methods for combining quality and efficiency performance measures: profiling the value of hospital care following acute myocardial infarction. Stat Med 27(9):1351-1370. https://doi.org/10.1002/sim.3082

6. Song Z, Safran DG, Landon BE, He Y, Mechanic RE, Day MP, Chernew ME (2012) The 'alternative quality contract,' based on a global budget, lowered medical spending and improved quality- supplemental materials. Health Aff (Millwood) 31(8):1885-1894

7. Kessler D, McClellan M (2001) The effects of hospital ownership on medical productivity. National Bureau of Economic Research, Cambridge

8. Wallace BC, Paul MJ, Sarkar U, Trikalinos TA, Dredze M (2014) A large-scale quantitative analysis of latent factors and sentiment in online doctor reviews. J Am Med Inform Assoc 21(6):1098-1103. https://doi.org/10.1136/amiajnl-2014-002711

9. Fenton JJ, Jerant AF, Bertakis KD (2012) The cost of satisfaction: national study of patient satisfaction, health care utilization, expenditures, and mortality. JAMA Intern Med 172(5):405-411 https://jamanetwork.com/journals/jamainternalmedicine/fullarticle/1108766

10. Cowing M, Davino-Ramaya CM, Ramaya K, Szmerekovsky J (2009) Health care delivery performance: service, outcomes, and resource stewardship. Perm J 13(4):72-78

11. Wright JD, Ananth CV, Tergas Al et al (2014) An economic analysis of robotically assisted hysterectomy. Obstet Gynecol 123:1038-1048

12. Wright JD, Ananth CV, Lewin SN et al (2013) Robotically assisted vs laparoscopic hysterectomy among women with benign gynecologic disease. JAMA 309(7):689-698

13. Andryjowicz $E$, Wray $T$ (2011) Regional expansion of minimally invasive surgery for hysterectomy: implementation and methodology in a large multispecialty group. Perm J 15(4):42-46

14. Sinha R, Sundaram M, Nikam YA, Hegde A, Mahajan C (2008) Total laparoscopic hysterectomy with earlier uterine artery ligation. JMIG 15(3): 355-359. https://doi.org/10.1016/j.jmig.2008.01.012

15. Poojari VG, Bhat W, Bhat R (2014) Total laparoscopic hysterectomy with prior uterine artery ligation at its origin. Int J Reprod Med:1-4. https://doi.org/10.1155/2014/420926

16. Lii SJ, Becker SF, Danilyants NE, Mackoul PJ (2010) A novel approach to total laparoscopic hysterectomy using only two $5 \mathrm{~mm}$ ports: initial clinical experience. JMIG 17:S87. https://doi.org/10.1016/j.jmig.2010.08.381

17. Elixhauser A, Steiner C, Harris DR, Coffey RM (1998) Comorbidity measures for use with administrative data. Med Care 36:8-27

18. Pelosi MA II, Pelosi MA III (2003) Pelosi minilaparotomy hysterectomy: effective alternative to laparoscopy and laparotomy. OBG Manag 15(4):16-33

19. Sokol DK, Wilson J (2008) What is a surgical complication? World J Surg 32(6):942-944. https://doi.org/10.1007/s00268-008-9471-6

20. Barnett JC, Judd JP, Wu JM, Scales CD Jr, Myers ER, Havrilesky LJ (2010) Cost comparison among robotic, laparoscopic, and open hysterectomy for endometrial cancer. Obstet Gynecol 116:685-693

21. Frick KD (2009) Microcosting quantity data collection methods. Med Care 47:S76-S81. https://doi.org/10.1097/MLR.0b013e31819bc064
22. Tan SS, Rutten FF, van Ineveld BM, Redekop WK, Hakkaart-van Roijen L (2009) Comparing methodologies for the cost estimation of hospital services. Eur J Health Econ 10:39-45. https://doi.org/10.1007/s10198-008-0101-x

23. Hospital Consumer Assessment of Healthcare Providers and Systems. https:// www.hcahpsonline.org/en/survey-instruments/. Accessed 11 Feb 2019

24. Pitter MC, Simmonds C, Seshadri-Kreaden U, Hubert HB (2014) The impact of different surgical modalities for hysterectomy on satisfaction and patient reported outcomes. Interact J Med Res 3:e11. https://doi.org/10.2196/ijmr.3160

25. Sarlos D, Kots L, Stevanovic N, Schaer G (2010) Robotic hysterectomy versus conventional laparoscopic hysterectomy: outcome and cost analyses of a matched casecontrol study. Eur J Obstet Gynecol Reprod Biol 150:92-96

26. Pasic RP, Rizzo JA, Fang H, Ross S, Moore M, Gunnarsson C (2010) Comparing robot-assisted with conventional laparoscopic hysterectomy: impact on cost and clinical outcomes. JMIG 17(6):730738. https://doi.org/10.1016/j.jmig.2010.06.009

27. Hikmet N, Chenb SK (2003) An investigation into low mail survey response rates of information technology users in health care organizations. Int J Med Inform 72(1-3):29-34

28. Fenton JJ, Jerant AF, Bertakis KD (2012) The cost of satisfaction; a national study of patient satisfaction, health care utilization, expenditures, and mortality. Arch Intern Med 172(5):405-411

29. Callahan D (2008) Health care costs and medical technology. In: Crowley M (ed) From birth to death and bench to clinic: the Hastings center bioethics briefing book for journalists, policymakers and campaigns. The Hastings Center, Garrison https:/www.thehastingscenter.org/wp-content/uploads/Health-Care-CostsBB17.pdf. Accessed 6 May 2015

30. The Affordable Care Act and trends in health care spending (2013). https://obamawhitehouse.archives.gov/sites/default/files/docs/fact sheet implementing_the_affordable_care_act_from_the_erp_2013_final1.pdf. Accessed 11 Feb 2019

31. Wieand E, Lagrew DC (2017) Value-based payment: what does it mean, and how can ObGyns get out ahead? OBG Manag 30(1):17-19 25-26

32. Madueke-Laveaux OS, Advincula AP (2017) Robot-assisted laparoscopy in benign gynecology: advantageous device or controversial gimmick? Best Pract Res Clin Obstet Gynaecol 45:2-6. https://doi.org/10.1016/j.bpobgyn.2017.09.011

33. Nieboer TE, Johnson N, Lethaby A et al (2009) Surgical approach to hysterectomy for benign gynaecological disease. Cochrane Database Syst Rev 3:CD003677. https://doi.org/10.1002/14651858.CD003677

34. Boyd LR, Novetsky AP, Curtin JP (2010) Effect of surgical volume on route of hysterectomy and short-term morbidity. Obstet Gynecol 116:909-915

35. Twijnstra ARH, Kolkman W, Trimbos-Kemper GCM, Jansen FW (2010) Implementation of advanced laparoscopic surgery in gynecology: national overview of trends. JMIG 17:487-492

36. Shepherd JP, Carter-Brooks CM, Kantartzis KL, Lee T, Bonidie MJ (2017) The impact of individual surgeon volume on hysterectomy costs. JSLS 21(1): e2016.00112. https://doi.org/10.4293/JSLS.2016.00112

37. Choosing the route of hysterectomy for benign disease: Committee Opinion No. 701 (2017) American College of Obstetricians and Gynecologists. Obstet Gynecol 129:155-159

38. SGO Clinical Practical Statement: Salpingectomy for ovarian cancer prevention. Available at: https://www.sgo.org/clinical-practice/guidelines/ sgo-clinical-practicestatement-salpingectomy-for-ovarian-cancer-prevention/. Accessed 27 Sept 2018

39. Robert M, Cenaiko D, Sepandj J, Iwanicki S (2015) Success and complications of salpingectomy at the time of vaginal hysterectomy. JMIG 22(5):864-869. https://doi.org/10.1016/j.jmig.2015.04.012

40. Burkett D, Horwitz J, Kennedy V, Murphy D, Graziano S, Kenton K (2011) Assessing current trends in resident hysterectomy training. Female Pelvic Med Reconstr Surg 17(5):210-214

41. Vree FE, Cohen SL, Chavan N, Einarsson JI (2014) The impact of surgeon volume on perioperative outcomes in hysterectomy. JSLS 18(2):171-181. https://doi.org/10.4293/108680813X13753907291594

42. Gold MR, Siegel JE, Russell LB, Weinstein MC (1996) Cost-effectiveness in health and medicine. Oxford University Press, New York 